Bundesgesundheitsbl 2018 $\cdot 61: 1196-1204$ https://doi.org/10.1007/s00103-018-2782-3 c) Springer-Verlag GmbH Deutschland, ein Teil von Springer Nature 2018

\section{Einleitung}

Die Internationalen Gesundheitsvorschriften (2005) (IGV) haben durch das „Gesetz zu den Internationalen Gesundheitsvorschriften (2005) (IGV) vom 23. Mai 2005" vom 20. Juli 2007 (Bundesgesetzblatt II, Seite 930) in Deutschland die Geltung eines Bundesgesetzes erlangt [1].

Artikel 20 Absatz 1 der IGV verpflichtet Deutschland als IGV-Vertragsstaat u. a. Häfen zu benennen, welche die in Anlage 1 der IGV vorgesehenen Kernkapazitäten zu schaffen und vorzuhalten haben. Artikel 19 Satz 1 Buchstabe a der IGV verpflichtet die Vertragsstaaten, dafür Sorge zu tragen, dass die in Anlage 1 der IGV unter für benannte Häfen beschriebenen Kernkapazitäten binnen fünf Jahren nach Inkrafttreten der IGV geschaffen werden. Die genannten Kernkapazitäten sollen die Vertragsstaaten gemäß Artikel 13 Absatz 1 Satz 1 IGV in die Lage versetzen, umgehend und wirksam auf Gefahren für die öffentliche Gesundheit und gesundheitliche Notlagen von internationaler Tragweite (GNIT) zu reagieren.

Das „Gesetz zur Durchführung der Internationalen Gesundheitsvorschriften (2005) (IGV-DG)“ [2] enthält dazu nähere Umsetzungsvorschriften. In Deutschland müssen an den Häfen der Städte Bremen

\title{
Kernkapazitäten von gemäß Art. 20 Absatz 1 IGV benannten Seehäfen für den Bereich der übertragbaren Krankheiten zur Durchführung der internationalen Gesundheitsvorschriften (2005; IGV) in Deutschland
}

\section{Empfehlung des Robert Koch- Institutes nach Anhörung der obersten Landesgesundheitsbehörden}

und Bremerhaven, Hamburg, Kiel, Rostock und am Jade-Weser-Port in Wilhelmshaven seit dem 15. Juni 2012 die in Anlage 1 Teil B IGV aufgeführten Kapazitäten zum Schutz der öffentlichen Gesundheit vorhanden sein ( $\$ 13$ Absatz 1 IGV-DG). Darüber hinaus können die zuständigen obersten Landesgesundheitsbehörden weitere Häfen bestimmen $(\$ 13$ Absatz 2 IGV-DG).

Die zuständige oberste Landesgesundheitsbehörde bestimmt unter Berücksichtigung des regelmäßigen Passagier- und Frachtaufkommens im Einzelnen Art und Umfang der Kapazitäten nach Anlage 1 Teil B IGV, die an dem jeweiligen den Hafen vorhanden sein müssen. Es müssen mindestens die Anforderungen der Anlage 1 Teil B IGV erfüllt sein ( $\$ 13$ Absatz 4 IGV-DG).

Da Anlage 1 Teil B der IGV lediglich in allgemeiner Weise beschreibt, welche Kernkapazitäten an den Häfen vorhanden sein müssen, hat das Robert KochInstitut gemäß $\$ 13$ Absatz 3 IGV-DG die Aufgabe, für den Bereich der übertragbaren Krankheiten eine Empfehlung zu den Kapazitäten gemäß Anlage 1 Teil B IGV abzugeben. Die vorliegende Empfehlung des Robert Koch-Institutes ist an die obersten Landesgesundheitsbehörden gerichtet. Sie hat als Empfehlung einen nicht verbindlichen Charakter. Sie dient dazu, die Kernkapazitäten systematisch darzustellen und die obersten Lan-

\begin{tabular}{|c|c|}
\hline \multicolumn{2}{|c|}{ Abkürzungen } \\
\hline $24 / 7$ & $\begin{array}{l}24 \text { Stunden am Tag/7 Tage die } \\
\text { Woche }\end{array}$ \\
\hline AkKü & $\begin{array}{l}\text { Arbeitskreis der Küstenländer } \\
\text { für Schiffshygiene }\end{array}$ \\
\hline$B G B I . I$ & Bundesgesetzblatt Teil I \\
\hline$B G B I . I I$ & Bundesgesetzblatt Teil II \\
\hline ggf. & gegebenenfalls \\
\hline GNIT & $\begin{array}{l}\text { Gesundheitliche Notlage } \\
\text { Internationaler Tragweite }\end{array}$ \\
\hline$H \ddot{D}$ & Hafenärztlicher Dienst \\
\hline IfSG & Infektionsschutzgesetz \\
\hline IGV & $\begin{array}{l}\text { Internationale Gesundheitsvor- } \\
\text { schriften (2005) }\end{array}$ \\
\hline$I G V-D G$ & $\begin{array}{l}\text { Gesetz zur Durchführung der } \\
\text { Internationalen Gesundheits- } \\
\text { vorschriften (2005) }\end{array}$ \\
\hline$M A C$ & Medical Assessment Center \\
\hline$M D$ & Medizinischer Dienst \\
\hline$O ̈ G D$ & Öffentlicher Gesundheitsdienst \\
\hline PLF & $\begin{array}{l}\text { Passenger Locator Form } \\
\text { (Aussteigekarte) }\end{array}$ \\
\hline$P S A$ & Persönliche Schutzausrüstung \\
\hline u. $a$. & unter anderem \\
\hline WHO & Weltgesundheitsorganisation \\
\hline
\end{tabular}




\begin{tabular}{|c|c|c|}
\hline \multicolumn{3}{|l|}{ Inhaltsverzeichnis } \\
\hline Abkürzungsverzeichnis & & 1.7 Kontrolle von Vektoren und Reservoiren \\
\hline Einleitung & & 1.7.1 Personal \\
\hline Hinweise & & 1.7.2 Ausrüstung \\
\hline Vorbemerkung & & 1.7.3 Maßnahmen \\
\hline 1 Jederzeit geforderte Kernkapazitäten & 2 & Kernkapazitäten für die Reaktion auf \\
\hline 1.1 Kommunikation & & Ereignisse, die eine gesundheitliche \\
\hline 1.1.1 Kommunikationsverfahren & & Notlage internationaler Tragweite (GNIT) \\
\hline 1.1.2 Kommunikationsinfrastruktur & & darstellen können \\
\hline und -sicherstellung & & 2.1 Reaktion auf Ereignisse, die GNIT \\
\hline 1.2 Personelle Kapazität des HÄD (ÖGD) & & darstellen können \\
\hline 1.3 Medizinische Dienste einschließlich & & 2.1.1 Kommunikation \\
\hline Diagnoseeinrichtungen & & 2.1.2 Allgemeine Hilfeleistungen bzw. \\
\hline 1.3.1 Schlüsselinformationen & & Unterstützungen \\
\hline 1.3.2 Personal & & 2.1.3 Räumlichkeiten bzw. Standort \\
\hline $\begin{array}{l}\text { a. Einsatzverantwortliche Person } \\
\text { des HÄD (ÖGD) für medizinische }\end{array}$ & & $\begin{array}{l}\text { für einen Einsatzraum für den HÄD } \\
\text { (ÖGD) }\end{array}$ \\
\hline Lagen & & 2.1.4 Notfallplan bzw. Standardarbeits- \\
\hline b. Koordinierende Ansprechperson & & anweisungen \\
\hline für den HÄD (ÖGD) im Hafen & & 2.2 Untersuchung und Versorgung von \\
\hline c. Medizinisches Personal & & betroffenen Reisenden oder Tieren \\
\hline $\begin{array}{l}\text { d. Lageabhängige Erweiterbarkeit } \\
\text { des Personals }\end{array}$ & & $\begin{array}{l}\text { 2.2.1 Untersuchung und Versorgung } \\
\text { von betroffenen Reisenden }\end{array}$ \\
\hline 1.3.3 Ausrüstung & & a. Personal \\
\hline 1.3.4 Räumlichkeiten & & b. Ausrüstung \\
\hline a. Berechtigter Zugang zum Hafen & & 2.2.2 Untersuchung und Versorgung \\
\hline b. Erste medizinische Bewertung & & von betroffenen Tieren \\
\hline $\begin{array}{l}\text { am Schiff } \\
\text { c. Räumlichkeiten bzw. Standort }\end{array}$ & & $\begin{array}{l}\text { a. Personal } \\
\text { b. Räumlich }\end{array}$ \\
\hline $\begin{array}{l}\text { c. Räumlichkeiten bzw. Standort } \\
\text { zur Lagerung medizinischer }\end{array}$ & & $\begin{array}{l}\text { b. Raumlichkeiten } \\
\text { 2.3 Räumlichkeiten für die Befragung }\end{array}$ \\
\hline Ausrüstung im Terminalbereich & & verdächtiger oder betroffener Personen \\
\hline d. Medizinische Dienste außerhalb & & 2.3.1 Personal \\
\hline des Hafens & & 2.3.2 Räumlichkeiten \\
\hline 1.4 Transport erkrankter Personen & & 2.3.3 Ausrüstung \\
\hline 1.4.1 Personal & & 2.4 Quarantäne verdächtiger Reisender \\
\hline 1.4.2 Ausrüstung & & 2.5 Durchführung empfohlener Maßnah- \\
\hline 1.5 Gewährleistung einer sicheren & & men \\
\hline Umgebung für Personen im Hafen & & 2.5.1 Personal \\
\hline 1.5.1 Personal & & 2.5.2 Räumlichkeiten \\
\hline 1.5.2 Ausrüstung & & 2.6 Medizinische Ein- und Ausreisekontrolle \\
\hline 1.5.3 Trinkwasserversorgung & & 2.6.1 Personal \\
\hline 1.5.4 Speiseräume bzw. Einrichtungen & & 2.6.2 Organisatorische Abwicklung \\
\hline der Bordverpflegung & & 2.7 Transfer von Reisenden, die möglicher- \\
\hline 1.5.5 Öffentliche Waschräume & & weise infiziert oder verseucht sind \\
\hline 1.5.6 Entsorgung von festen und & & 2.7.1 Personal \\
\hline flüssigen Abfällen & & 2.7.2 Ausrüstung \\
\hline 1.5.7 Leichen & 3 & Kontaktpersonen-Ermittlung \\
\hline 1.5.8 Tierkadaver & 4 & Ausbildung und Inübunghaltung \\
\hline $\begin{array}{l}\text { 1.6 Überprüfung von Beförderungsmitteln } \\
\text { 1.6.1 Personal }\end{array}$ & 5 & $\begin{array}{l}\text { Erfahrungsaustausch und Fortentwicklung } \\
\text { der Kernkapazitäten }\end{array}$ \\
\hline 1.6.2 Ausrüstung & 6 & Referenzen \\
\hline
\end{tabular}

desgesundheitsbehörden bei ihrer Entscheidung gemäß \$13 Absatz 4 IGV-DG zu unterstützen. Die zuständigen obersten Landesgesundheitsbehörden wurden zu der Empfehlung angehört.

Da es sich bei den gemäß $\$ 13$ Absatz 1 oder 2 IGV-DG benannten Häfen derzeit ausschließlich um Seehäfen handelt, bezieht sich vorliegende Empfehlung ausschließlich auf den Seeschiffsverkehr und nicht auf den Binnenschiffsverkehr.
Soweit diese Empfehlung keine anderen Angaben enthält, wird für die quantitative Bemessung der Kernkapazitäten im Folgenden davon ausgegangen, dass die Passagier- und Besatzungsanzahl des größten planmäßig am benannten Hafen einlaufenden Schiffes gehandhabt werden kann.

Der Anwendungsbereich der IGV erstreckt sich auf Krankheiten „ungeachtet des Ursprungs oder der Quelle“. Dementsprechend beziehen sich die erforder- lichen Kernkapazitäten auf Gesundheitsgefahren aufgrund von übertragbaren Krankheiten und darüber hinaus auch auf für einen Hafen relevante Gesundheitsgefahren aufgrund von chemischen und radionuklearen Agenzien. Die vorliegende Empfehlung bezieht sich gemäß $\$ 13 \mathrm{Ab}$ satz 3 IGV-DG nur auf den Bereich der übertragbaren Krankheiten.

Die Verwendung von Begriffen entspricht den Definitionen des IGV-DG und der IGV sowie des Infektionsschutzgesetzes (IfSG). Es gilt zu beachten, dass der Hafenärztliche Dienst (HÄD) Teil des Gesundheitsamtes (GA) sein kann und damit dem Öffentlichen Gesundheitsdienst (ÖGD) zuzuordnen ist.

Der ÖGD ist - wie die öffentliche Verwaltung insgesamt - zur Beachtung des Rechtsgrundsatzes der Verhältnismäßigkeit verpflichtet. Hiernach müssen alle Maßnahmen des öffentlichen Gesundheitsdienstes, die in Grundrechte Privater eingreifen, zur Erreichung ihres Zwecks geeignet und erforderlich sein und der besonderen Bedeutung der ggf. betroffenen Grundrechte gerecht werden (Abwägung der grundrechtlichen Schutzgüter mit den durch den Eingriff verfolgten öffentlichen Zwecken).

Die vorliegende Empfehlung betrifft Kapazitäten, die von dem Land und dem Hafenbetreiber an dem Hafen vorsorglich geschaffen und unterhalten werden sollen. Davon unberührt bleibt die Befugnis der zuständigen Behörden, bei einer konkreten Gefahr im Einzelfall Anordnungen zur Gefahrenabwehr etwa nach $\$ 16$ oder $\$ 28$ des Infektionsschutzgesetzes oder auf der Grundlage anderer Fachgesetze oder des Polizeigesetzes des jeweiligen Bundeslandes zu treffen. Bei diesen Maßnahmen können zur Abwehr der Gefahr u. U. auch Reeder, sonstige Beförderungsunternehmen, Terminalbetreiber oder andere unmittelbar in Anspruch genommen werden; es gelten dann die Grundsätze des allgemeinen Ordnungsrechts über die Störerauswahl und die - wenn zur Abwehr einer gegenwärtigen, erheblichen und nicht anders abwendbaren Gefahr erforderlich - Inanspruchnahme von Nichtstörern. 


\section{Hinweise}

- Gemäß $\$ 13$ Absatz 4 IGV-DG bestimmt die zuständige oberste Landesgesundheitsbehörde die konkreten Kernkapazitäten, die am jeweiligen Hafen vorhanden sein müssen. Dazu sind soweit erforderlich vor Ort administrative Vorkehrungen und Vereinbarungen zu treffen. Diese sollten unter Koordination des HÄD (ÖGD) erstellt werden.

- Das Vorgehen im Ereignisfall ist in den Notfallplänen der designierten Häfen zu beschreiben. Die Länder stimmen sich hierzu im Arbeitskreis der Küstenländer (AkKü) für Schiffshygiene ab.

\section{Vorbemerkung}

Die Gliederung der Anlage 1 Teil B der IGV unterscheidet zwischen folgenden Kernkapazitäten, um gemäß Artikel 13 der IGV umgehend und wirksam auf Gefahren für die öffentliche Gesundheit und GNIT zu reagieren:

- Kernkapazitäten, die jederzeit gefordert werden (Anlage 1 Teil B Absatz 1 der IGV; hierzu nachfolgend unter $1)$; und

- Kernkapazitäten, die zusätzlich für die Reaktion auf Ereignisse gefordert werden, die eine gesundheitliche Notlage von internationaler Tragweite darstellen können (Anlage 1 Teil B Absatz 2 der IGV; hierzu nachfolgend unter 2).

Die in dieser Empfehlung angesprochene Eigenschaft eines Hafens als „benannter" Hafen mit Kernkapazitäten für den Gesundheitsschutz gemäß Artikel 20 Absatz 1 IGV ist grundsätzlich zu unterscheiden von der Eigenschaft eines Hafens als „befugter" Hafen gemäß Artikel 20 Absatz 3 IGV, welcher Bescheinigungen über die Schiffshygiene ausstellen oder verlängern darf. Aufgrund der von benannten Häfen zu verlangenden Fähigkeiten kommen als benannte Häfen allerdings nur Häfen gemäß Artikel 20 Absatz 3 Buchstabe a IGV in Betracht, deren HÄD dazu befugt ist, die Bescheinigung über die Durchführung von Schiffshygienemaßnahmen gemäß Artikel 39 Absatz 5 IGV auszustellen (vgl. \$13 Absatz 2 Satz 2 IGV-DG).
Die an die Kernkapazitäten zu stellenden Anforderungen werden nachfolgend systematisch konkretisiert.

\section{Jederzeit geforderte Kernkapazitäten}

Die Kapazitäten, die ,jederzeit“ gefordert sind, sind in Anlage 1 Teil B Absatz 1 der IGV genannt. Es handelt sich hierbei um Kernkapazitäten, die im Routinebetrieb vorhanden sein sollten.

\subsection{Kommunikation}

\section{Empfehlung:}

\subsubsection{Kommunikationsverfahren}

Administrative Vorkehrungen und Vereinbarungen liegen vor, für ein definiertes, dokumentiertes und bei allen Beteiligten bekanntes und trainiertes Verfahren der Kommunikation

- für die Übermittlung einer Seegesundheitserklärung rechtzeitig vor Einlaufen in den Hafen, unter Nutzung etablierter Meldestrukturen. Die Implementierung des Meldeverfahrens muss gemäß $\$ 15 \mathrm{Ab}$ satz 1 elektronisch nach Maßgabe des Seeschifffahrt-Meldeportal-Gesetzes (Umsetzung des Single Window Verfahrens der EU-Richtlinie 2010/65/ EU) erfolgen [2, 3]. Die Nachmeldung oder Korrektur von Angaben hat unverzüglich nach deren Vorliegen zu erfolgen und es muss sichergestellt werden, dass diese Angaben den HÄD (ÖGD) unverzüglich erreichen;

- für die Erteilung bzw. Übermittlung der freien Verkehrserlaubnis an das Schiff oder den Betreiber des Schiffes. Wenn die freie Verkehrserlaubnis verweigert wird, ist zusätzlich die zuständige Hafenaufsicht zu beteiligen;

- unter allen einsatzbeteiligten und -betroffenen Stellen im Hafen wie HÄD (ÖGD), Hafenaufsicht, Hafen- und Terminalbetreibern, Betreibern von Beförderungsmitteln, Dienstleistungsunternehmen und weiteren für die Notfallplanung relevanten Grenzübergangsstellen, Gesundheitseinrichtungen und -diensten und anderen relevanten Einrichtungen und Behörden wie Verkehrszentralen;
- mit weiteren Experten des ÖGD zur raschen Entscheidungsfindung, Risikobewertung und Implementierung von Maßnahmen zur Begrenzung und Kontrolle des Ereignisses;

- mit medizinischem Personal an Bord, sofern vorhanden, bzw. mit dem Schiffsführer;

- mit der Bundes- bzw. Wasserschutzpolizei und mit dem Zoll;

- mit den Reisenden und auf diese wartenden Personen bzw. Angehörigen bezüglich gesundheitsbezogener Informationen;

- mit anderen relevanten nationalen und internationalen Häfen, um diese über festgestellte Infektions- oder Verseuchungsquellen an Bord und ggf. über durchgeführte oder noch durchzuführende Gesundheitsmaßnahmen zu informieren.

\subsubsection{Kommunikationsinfrastruktur} und -sicherstellung

- Der HÄD (ÖGD) hält für den Eigenbedarf eine Ausstattung mit zeitgemäßen Kommunikationsmitteln vor und stellt deren Einsatzfähigkeit sicher.

- Der HÄD (ÖGD) stellt sicher, dass

- er die technischen und personellen Ressourcen schafft, um die Meldungen der Schiffsführer vor Einlaufen des Schiffes entgegennehmen und bewerten zu können;

- er oder seine Beauftragten die freie Verkehrserlaubnis erteilen können.

\subsection{Personelle Kapazität des HÄD} (ÖGD)

- Die Personalkapazität des HÄD (ÖGD) orientiert sich am Schiffsaufkommen, siehe $\$ 13$ IGV-DG [4], Mindestanforderungen sind:

- die jederzeitige Reaktion auf Ereignisse und Meldungen;

- die Möglichkeit, die freie Verkehrserlaubnis für Passagierschiffe jederzeit vor Ort zu erteilen; und

- die Möglichkeit, täglich Schiffshygienebescheinigungen ausstellen und ggf. damit verbundene Schiffshygienemaßnahmen durchführen zu können. 


\subsection{Medizinische \\ Dienste einschließlich \\ Diagnoseeinrichtungen}

Gemäß Anlage 1 Teil B Absatz 1 Buchstabe a der IGV wird die Kapazität gefordert, jederzeit den Zugang

1. zu geeigneten medizinischen Diensten einschließlich Diagnoseeinrichtungen, die so gelegen sind, dass eine sofortige Untersuchung und Versorgung erkrankter Reisender ermöglicht wird sowie

2. zu geeignetem Personal, geeigneter Ausrüstung und geeigneten Räumlichkeiten sicherzustellen.

\section{Empfehlung:}

\subsubsection{Schlüsselinformationen}

Schlüsselinformationen betreffend medizinische Dienste einschließlich Diagnoseeinrichtungen liegen im Hafen vor:

- Eine Liste mit Diensten und relevanten Einrichtungen, einschließlich der Namen der verantwortlichen Ansprechpersonen und Schlüsselinformationen (Adresse, Telefonnummer, Fax, E-Mail, ggf. Entfernung vom Hafen und Karte mit Wegbeschreibung) ist erstellt und wird erhalten und aktualisiert, verteilt und regelmäßig auf Richtigkeit überprüft.

- Diese Liste ist jederzeit aktuell und zugänglich für das gesamte relevante Personal.

\subsubsection{Personal}

a. Einsatzverantwortliche Person des HÄD (ÖGD) für medizinische Lagen Administrative Vorkehrungen und Vereinbarungen liegen vor, für

- 24 Stunden am Tag/7 Tage die Woche (24/7) Erreichbarkeit bzw. Verfügbarkeit, wie jeweils angemessen, eines einsatzverantwortlichen Arztes des HÄD (ÖGD). Diese Person schätzt die Lage ein und entscheidet zeitnah über das weitere Vorgehen und kommuniziert alle relevanten Ereignisse zeitgerecht.

\section{b. Koordinierende Ansprechperson für den HÄD (ÖGD) im Hafen}

Administrative Vorkehrungen und Vereinbarungen liegen vor, für
- 24/7 Erreichbarkeit bzw. Verfügbarkeit, wie jeweils angemessen, einer koordinierenden Ansprechperson im Hafen mit Kenntnissen der Strukturen und Abläufe des Hafens zur Umsetzung der von der einsatzverantwortlichen Person des HÄD (ÖGD) (1.3.2] a.) angeordneten Maßnahmen. Diese koordinierende Ansprechperson wird von der zuständigen Hafenaufsicht benannt ( $\$ 13$ Absatz 9 Satz 2 IGV-DG).

\section{c. Medizinisches Personal}

Administrative Vorkehrungen und Vereinbarungen liegen vor, für

- 24/7 Zugang zu ausgebildeten und benannten Ärzten und Assistenzpersonal, welche im Auftrag des HÄD (ÖGD) arbeiten, zur zeitnahen Untersuchung, Befragung, medizinischen Versorgung und ggf. Absonderung von betroffenen Personen (erkrankt oder ansteckungsverdächtig).

- Mindestens: 1 Arzt und 1 medizinischer Assistent.

- Reaktionszeit: rechtzeitig verfügbar bei Anlegen des Schiffes bei regelhaftem Eingang einer Seegesundheitserklärung mit mindestens einer bejahten Frage über die Gesundheit in der Seegesundheitserklärung.

\section{d. Lageabhängige Erweiterbarkeit des Personals}

Administrative Vorkehrungen und Vereinbarungen liegen vor, für

- Regelungen zur lageabhängigen personellen Erweiterbarkeit, z. B. mit

- HÄD (ÖGD)-Personal;

- Ärzten und medizinischem Assistenzpersonal; und

- Personal des Rettungsdienstes.

\subsubsection{Ausrüstung}

Administrative Vorkehrungen und Vereinbarungen liegen vor, für

- Persönliche Schutzausrüstung (PSA) und Hilfsmittel zur Reaktion auf ein Gesundheitsrisiko;

- die lageabhängige Verfügbarkeit von medizinisch-technischer Ausrüstung zur Untersuchung, Erstversorgung bzw. Therapie und Prophylaxe (Präund Postexposition, Mund-Nasen-
Schutz), Probenentnahme und zum Probentransport; und

- Verpackungsmaterial zum Transport von Untersuchungsmaterial bzw. Proben in geeignete Diagnoseeinrichtungen bzw. Labore gemäß den rechtlichen Bestimmungen.

\subsubsection{Räumlichkeiten}

\section{a. Berechtigter Zugang zum Hafen}

Administrative Vorkehrungen und Vereinbarungen liegen vor, für

- Gestattungen zum jederzeitigen, berechtigten Zugang des medizinischen Personals bzw. des HÄD (ÖGD) zu nicht allgemein zugänglichen bzw. zu sicherheitsempfindlichen Bereichen zur regelhaften Überwachung der Schiffs- und Hafenhygiene und Reaktion auf Ereignisse und Meldungen.

\section{b. Erste medizinische Bewertung am} Schiff

Administrative Vorkehrungen und Vereinbarungen liegen vor, für

- die Bestimmung eines im Bedarfsfall geeigneten Liegeplatzes mit Landzugang im jeweils betroffenen Hafen, unter Berücksichtigung der Anforderungen des Gesundheitsschutzes und der nautischen Bedingungen (wie Länge, Breite, Tiefgang, Art des Schiffes), s. auch $₫ 13$ Absatz 5 Satz 1 Nummer 1 IGV-DG;

- die Sicherstellung des uneingeschränkten Zugangs zu Schiffen durch medizinisches Personal und den HÄD (ÖGD);

- die Gewährleistung einer Zutrittsbeschränkung zum Schiff für nicht autorisierte Personen;

- die Gewährleistung der Zufahrt zum Schiffsliegeplatz für Fahrzeuge des Rettungsdienstes und der Feuerwehr, für Busse etc; und

- ein definiertes, dokumentiertes und bei allen Beteiligten bekanntes und trainiertes Verfahren

- zur Untersuchung und Versorgung von betroffenen Personen an Bord im Ereignisfall unter Berücksichtigung der Fortführung des Schiffsbetriebes;

- zur Absonderung von erkrankten Personen an Bord im Ereignisfall 
unter Berücksichtigung der Fortführung des Schiffsbetriebes; und

- zur Durchführung einer Kontaktpersonen-Ermittlung ( $\$ 17$ Absatz 3 IGV-DG) im Ereignisfall an Bord unter Berücksichtigung der Fortführung des Schiffsbetriebes.

c. Räumlichkeiten bzw. Standort zur Lagerung medizinischer Ausrüstung im Terminalbereich

Administrative Vorkehrungen und Vereinbarungen liegen vor, für

- die lageabhängige Nutzung von geeigneten Räumlichkeiten bzw. Bereitstellung eines geeigneten Standorts auf dem Terminalgelände zur Lagerung der erforderlichen Ausrüstung des HÄD (ÖGD) für die Befragung, Untersuchung und Versorgung von verdächtigen oder betroffenen Reisenden im Ereignisfall (s. auch $\$ 13$ Absatz 5 Satz 1 Nummer 2 IGV-DG).

\section{d. Medizinische Dienste außerhalb des} Hafens

Administrative Vorkehrungen und Vereinbarungen liegen vor, für

- die 24/7 gesicherte Aufnahme in geeignete medizinische Einrichtungen, ggf. Sonderisolierstation zur sofortigen Untersuchung und Versorgung erkrankter Personen.

\subsection{Transport erkrankter Personen}

Gemäß Anlage 1 Teil B Absatz 1 Buchstabe b der IGV wird die Kapazität gefordert, jederzeit den Zugang zu Ausrüstung und Personal für den Transport erkrankter Reisender zu geeigneten medizinischen Einrichtungen sicherzustellen.

Empfehlung:

\subsubsection{Personal}

Administrative Vorkehrungen und Vereinbarungen liegen vor, für

- den 24/7 Zugang zu ausgebildetem und benanntem Personal zur Sicherstellung eines angemessenen Transportes erkrankter Personen in geeignete medizinische Einrichtungen.

\subsubsection{Ausrüstung}

Administrative Vorkehrungen und Vereinbarungen liegen vor, für

- die Vorhaltung der erforderlichen persönlichen Schutzausrüstung; und

- den 24/7 Zugang zu Fahrzeugen für den sicheren und hygienischen Transport erkrankter Personen zu geeigneten medizinischen Einrichtungen.

\subsection{Gewährleistung einer sicheren Umgebung für Personen im Hafen}

Gemäß Anlage 1 Teil B Absatz 1 Buchstabe d der IGV wird die Kapazität gefordert, jederzeit je nach Bedarf durch Überprüfungsprogramme eine sichere Umgebung für Reisende zu gewährleisten, die Einrichtungen von Grenzübergangsstellen nutzen, darunter die Trinkwasserversorgung, Speiseräume, Einrichtungen der Bordverpflegung, öffentliche Waschräume, geeignete Entsorgungseinrichtungen für feste und flüssige Abfälle und andere potenzielle Risikobereiche.

Empfehlung (s. auch $\$ 13$ Absatz 5 Satz 1 Nummer 3 IGV-DG):

\subsubsection{Personal}

Administrative Vorkehrungen und Vereinbarungen liegen vor, für

- den Zugang zu ausgebildetem und benanntem Personal für die Überwachung der Hygiene und des Gesundheitsschutzes im Hafen.

\subsubsection{Ausrüstung}

Administrative Vorkehrungen und Vereinbarungen liegen vor, für

- die Vorhaltung der erforderlichen persönlichen Schutzausrüstung; und

- den Zugang zu erforderlicher Ausrüstung, welche die Einhaltung der allgemein anerkannten Regeln der Technik und der rechtlichen Bestimmungen gewährleistet (u. a. Hygiene, Trinkwasser, Abwasser, Abfallbeseitigung).

\subsubsection{Trinkwasserversorgung}

Administrative Vorkehrungen und Vereinbarungen liegen vor, für

- ein definiertes, dokumentiertes und bei allen Beteiligten bekanntes und trainiertes Verfahren der Bereitstellung von Trinkwasser, gemäß den rechtlichen Bestimmungen der Trinkwasserverordnung in der jeweils gültigen Fassung und den allgemein anerkannten Regeln der Technik.

\subsubsection{Speiseräume bzw.}

Einrichtungen der Bordverpflegung

Administrative Vorkehrungen und Ver-

einbarungen liegen vor, für

- ein definiertes, dokumentiertes und bei allen Beteiligten bekanntes und trainiertes Verfahren zur Gewährleistung einer sicheren Umgebung in Speiseräumen, wenn vorhanden, gemäß den rechtlichen Bestimmungen und den allgemein anerkannten Regeln der Technik.

\subsection{5 Öffentliche Waschräume}

Administrative Vorkehrungen und Vereinbarungen liegen vor, für

- ein definiertes, dokumentiertes und bei allen Beteiligten bekanntes und trainiertes Verfahren zur Gewährleistung einer sicheren Umgebung in öffentlichen Waschräumen, wenn vorhanden, gemäß den rechtlichen Bestimmungen und den allgemein anerkannten Regeln der Technik.

\subsubsection{Entsorgung von festen und flüssigen Abfällen}

Administrative Vorkehrungen und Vereinbarungen liegen vor, für

- ein definiertes, dokumentiertes und bei allen Beteiligten bekanntes und trainiertes Verfahren der Behandlung und Entsorgung von festen und flüssigen Abfällen insbesondere im Hinblick auf Abfälle, welche biologische Gefahren für die Gesundheit darstellen, gemäß den rechtlichen Bestimmungen und den allgemein anerkannten Regeln der Technik.

\subsubsection{Leichen}

Administrative Vorkehrungen und Vereinbarungen liegen vor, für

- ein definiertes, dokumentiertes und bei allen Beteiligten bekanntes und trainiertes Verfahren des Umgangs mit Leichen und Leichenteilen, gemäß den rechtlichen Bestimmungen und den allgemein anerkannten Regeln der Technik. 


\subsubsection{Tierkadaver}

Administrative Vorkehrungen und Vereinbarungen liegen vor, für

- ein definiertes, dokumentiertes und bei allen Beteiligten bekanntes und trainiertes Verfahren des Umgangs mit verstorbenen Tieren, gemäß den rechtlichen Bestimmungen und den allgemein anerkannten Regeln der Technik.

\section{6 Überprüfung von Beförderungsmitteln}

Gemäß Anlage 1 Teil B Absatz 1 Buchstabe $c$ der IGV wird die Kapazität gefordert, jederzeit ausgebildetes Personal für die Überprüfung von Beförderungsmitteln bereitzustellen.

\section{Empfehlung:}

\subsubsection{Personal}

Administrative Vorkehrungen und Vereinbarungen liegen vor, für

- den 24/7 Zugang zu ausgebildetem, regelmäßig geschultem und benanntem Personal für die Überprüfung von Beförderungsmitteln z. B. auf Kontamination.

\subsubsection{Ausrüstung}

Administrative Vorkehrungen und Vereinbarungen liegen vor, für

- die Vorhaltung der erforderlichen persönlichen Schutzausrüstung; und

- den Zugang zu erforderlicher Ausrüstung für die Überprüfung von Beförderungsmitteln.

\subsection{Kontrolle von Vektoren und Reservoiren}

Gemäß Anlage 1 Teil B Absatz 1 Buchstabe e der IGV wird die Kapazität gefordert, jederzeit soweit durchführbar ein Programm und ausgebildetes Personal für die Bekämpfung von Vektoren und Herden in und in der Nähe von Grenzübergangsstellen bereitzustellen.
Empfehlung:

(s. auch $\$ 13$ Abs. 5 Satz 1 Nummer 5 IGVDG):

\subsubsection{Personal}

Administrative Vorkehrungen und Vereinbarungen liegen vor, für

- den 24/7 Zugang zu ausgebildetem und qualifiziertem Personal zur Bekämpfung von Vektoren und Reservoiren.

\subsubsection{Ausrüstung}

Administrative Vorkehrungen und Vereinbarungen liegen vor, für

- die Vorhaltung der erforderlichen persönlichen Schutzausrüstung;

- den Zugang zu erforderlicher Ausrüstung für die Kontrolle von Vektoren und Reservoiren; und

- die regelmäßige Kontrolle der Ausrüstung (u. a. Haltbarkeit, Bestand) [4].

\subsubsection{Maßnahmen}

Administrative Vorkehrungen und Vereinbarungen liegen vor, für

- die Freihaltung des Hafengeländes einschließlich eines Umkreises von 400 Metern von Vektoren und Erregerreservoiren durch periodische Maßnahmen zur Prävention bzw. Bekämpfung von Vektoren und Erregerreservoiren in Gebäuden und auf dem Hafenareal.

\section{Kernkapazitäten für die Reaktion auf Ereignisse, die eine gesundheitliche Notlage internationaler Tragweite (GNIT) darstellen können}

Die Kernkapazitäten, die „für die Reaktion auf Ereignisse, die eine gesundheitliche Notlage von internationaler Tragweite darstellen können “ gefordert sind, sind in Anlage 1 Teil B Absatz 2 der IGV genannt. Es handelt sich hierbei um Kernkapazitäten, die im Falle des Auftretens gesundheitlicher Notlagen vorhanden sein sollten bzw. aktiviert werden können.

\subsection{Reaktion auf Ereignisse, die GNIT darstellen können}

Gemäß Anlage 1 Teil B Absatz 2 Buchstabe a der IGV wird die Kapazität gefordert, für die Reaktion auf mögliche GNIT-Er- eignisse eine angemessene Reaktion auf gesundheitliche Notlagen zu ermöglichen, indem ein Notfallplan für gesundheitliche Notlagen entwickelt und fortgeführt wird, einschließlich der Benennung eines Koordinators und von Anlaufstellen für relevante Grenzübergangsstellen, Gesundheitseinrichtungen und -dienste und andere Einrichtungen und Dienste.

\section{Empfehlung:}

\subsubsection{Kommunikation}

Administrative Vorkehrungen und Vereinbarungen liegen vor, für ein definiertes, dokumentiertes und bei allen Beteiligten bekanntes und trainiertes Verfahren - zur lageabhängigen Information von Reisenden:

- Erstellung von mehrsprachigen Informationsblättern und Plakaten in Abstimmung mit den zuständigen Behörden (z. B. Deutsch, Englisch, Französisch, Spanisch, Russisch).

- Bereitstellung der Information, u. a. durch Nutzung von Bildschirmen, Informationsflächen, Ständern für Informationsblätter.

- zur Abstimmung der Kommunikation nach außen:

- Einbeziehung betroffener Organisationen und Unternehmen, unter lageabhängiger Festlegung der $\mathrm{Fe}$ derführung in die behördliche Krisenkommunikationsstruktur.

\subsubsection{Allgemeine Hilfeleistungen bzw. Unterstützungen}

Vorkehrungen und Vereinbarungen liegen vor,

- zur Sicherstellung von allgemeinen Hilfeleistungen bzw. Unterstützung der betroffenen Personen und auf diese wartende Personen und Angehörige (u. a. Information, Hilfe bei der Weiterreise, Umbuchungen, Benachrichtigung von Angehörigen, Zugang zu Telekommunikation).

\subsubsection{Räumlichkeiten bzw. Standort für einen Einsatzraum für den HÄD (ÖGD)}

Administrative Vorkehrungen und Vereinbarungen liegen vor, für

- die Nutzung von geeigneten Räumlichkeiten bzw. einen geeigneten 
Standort für einen Einsatzraum für den HÄD (ÖGD).

\subsubsection{Notfallplan bzw. Standardarbeitsanweisungen}

Notfallplan (s. auch $\$ 13$ Absatz 5 Satz 1 Nummer 4 IGV-DG) bzw. Standardarbeitsanweisungen - in Abstimmung mit den zuständigen Gesundheitsbehörden liegen vor für

- eine Aufstockung der personellen Kapazitäten im Ereignisfall (siehe auch Punkt 1.3.2 d.) z. B. mit

- HÄD (ÖGD)-Personal,

- Ärzten und medizinischem Assistenzpersonal,

- Personal des Rettungsdienstes,

- Personal aus Hilfsorganisationen, und

- Personal aus Organisationen des Katastrophenschutzes;

- die Reaktionszeiten des benötigten Personals;

- abhängig von der Gefahrenlage die Anzahl des benötigten Personals pro Anzahl betroffener Personen und Stunde;

- eine Aufstockung der Ausrüstung im Ereignisfall (siehe Punkt 1.3.3), z. B. mit

- persönlicher Schutzausrüstung und Hilfsmitteln zur Reaktion auf ein Gesundheitsrisiko;

- lageabhängiger Verfügbarkeit von medizinisch-technischer Ausrüstung zur Untersuchung, Erstversorgung bzw. Therapie und Prä- und Postexpositionsprophylaxe (Medikamente und Impfstoffe), Infektionsminimierung (z. B. Mund-Nasen-Schutz), Probenentnahme und zum Probentransport; und

- Verpackungsmaterial zum Transport von Untersuchungsmaterial bzw. Proben in geeignete Diagnoseeinrichtungen bzw. Labore gemäß den rechtlichen Bestimmungen;

- die Anpassung bzw. Fortschreibung bestehender Notfallpläne in Abstimmung mit allen beteiligten Einrichtungen und Behörden;

- eine Überprüfung der Notfallplanung mindestens alle 2 Jahre (z. B. Planübung, Alarmierungsübung, Praxisübung);

- ein definiertes, dokumentiertes und bei allen Beteiligten bekanntes und trainiertes Verfahren zur Identifikation von ansteckungsverdächtigen oder erkrankten Personen, ihrer medizinischen, sozialen, organisatorischen Betreuung und Dokumentation;

- ein definiertes, dokumentiertes und bei allen Beteiligten bekanntes und trainiertes Verfahren von medizinische Ein- und Ausreisekontrollen, z. B. Farbkodierung; und

- ein definiertes, dokumentiertes und bei allen Beteiligten bekanntes und trainiertes Verfahren hinsichtlich des bevorrechtigten Zutritts des medizinischen bzw. veterinärmedizinischen Personals (insbesondere der Gesundheitsbehörden) zu sicherheitsbeschränkten Bereichen des Hafens. Es wird der zuständigen Aufsichtsbehörde empfohlen, unter Berücksichtigung der rechtlichen Bestimmungen, den regelmäßig im Hafen eingesetzten Mitarbeitern des HÄD (ÖGD) und deren mitgeführten Fahrzeugen bevorrechtigten Zugang in die sicherheitsrelevanten Bereiche des Hafens zu ermöglichen (Gestattung im Einsatzfall).

\subsection{Untersuchung und Versorgung von betroffenen Reisenden oder Tieren}

Gemäß Anlage 1 Teil B Absatz 2 Buchstabe b der IGV wird die Kapazität gefordert, für die Reaktion auf mögliche GNIT-Ereignisse die Untersuchung und Versorgung von betroffenen Reisenden oder Tieren sicherzustellen, indem Vereinbarungen mit medizinischen und tiermedizinischen Einrichtungen vor Ort über ihre Absonderung, ihre Behandlung sowie über etwa erforderliche andere unterstützende Leistungen getroffen werden,

\subsubsection{Untersuchung und Versorgung von betroffenen Reisenden}

Empfehlung:

\section{a. Personal}

Administrative Vorkehrungen und Vereinbarungen liegen vor, für

- die lageabhängige Anpassung der Anzahl des eingesetzten Personals.

\section{b. Ausrüstung}

Administrative Vorkehrungen und Vereinbarungen liegen vor, für

- die lageabhängige Anpassung der Anzahl und Art der eingesetzten Ausrüstung.

\subsubsection{Untersuchung und} Versorgung von betroffenen Tieren Empfehlung, sobald im jeweiligen Hafen Lebendtiertransport durchgeführt wird:

\section{a. Personal}

Administrative Vorkehrungen und Vereinbarungen liegen vor, für

- die 24/7 Erreichbarkeit der zuständigen Veterinärbehörde; und

- die fachliche Beratung bzw. Entscheidung der Veterinärbehörde über das weitere Vorgehen.

\section{b. Räumlichkeiten}

Administrative Vorkehrungen und Vereinbarungen liegen vor, für

- den Zugang zu bzw. die Bereitstellung eines Absonderungs- bzw. Untersuchungsraums für erkrankte Tiere;

- den Zugang zu einer tiermedizinischen Einrichtung (i.d. R. Tierklinik); und

- ein definiertes, dokumentiertes Verfahren der Kommunikation mit den veterinär-medizinischen Kompetenzzentren sowie der Zuweisung, des Transportes und Übergabe der erkrankten Tiere.

\subsection{Räumlichkeiten für die} Befragung verdächtiger oder betroffener Personen

Gemäß Anlage 1 Teil B Absatz 2 Buchstabe c der IGV wird die Kapazität gefordert, für die Reaktion auf mögliche GNIT-Ereignisse geeignete, von anderen Reisenden getrennte Räumlichkeiten für die Befragung verdächtiger oder betroffener Personen bereitzustellen.

\section{Empfehlung:}

\subsubsection{Personal}

Administrative Vorkehrungen und Vereinbarungen liegen vor, für

- die lageabhängige Anpassung der Anzahl des eingesetzten Personals. 


\subsubsection{Räumlichkeiten}

Zur Befragung verdächtiger oder betroffener Personen können im Ereignisfall in Abstimmung mit der Schiffsführung Räumlichkeiten an Bord eines Schiffes genutzt werden. Dabei ist die Fortführung des Schiffsbetriebes, soweit möglich, zu berücksichtigen.

Darüber hinaus müssen Administrative Vorkehrungen und Vereinbarungen vorliegen, für

- die Nutzung von bestehenden Räumlichkeiten oder die Bereitstellung von Flächen für die Schaffung von Räumlichkeiten in der Nähe des Liegeplatzes.

\subsubsection{Ausrüstung}

Administrative Vorkehrungen und Vereinbarungen liegen vor, für

- die lageabhängige Anpassung der Anzahl und Art der eingesetzten Ausrüstung.

\subsection{Quarantäne verdächtiger Reisender}

Gemäß Anlage 1 B. Absatz 2 Buchstabe d der IGV wird die Kapazität gefordert, für die Reaktion auf mögliche GNIT-Ereignisse für die Untersuchung und nötigenfalls für die Quarantäne verdächtiger Reisender zu sorgen, vorzugsweise in von der Grenzübergangsstelle entfernt gelegenen Einrichtungen.

\section{Empfehlung:}

Administrative Vorkehrungen und Vereinbarungen liegen vor, für ein definiertes, dokumentiertes und bei allen Beteiligten bekanntes und trainiertes Verfahren zur lageabhängigen

- Quarantäne verdächtiger Reisender im Ereignisfall an Bord eines Schiffes unter Berücksichtigung der Fortführung des Schiffsbetriebes; und

- Nutzung der in den Notfallplänen vorgesehenen funktionsfähigen Einrichtungen.

\subsection{Durchführung empfohlener Maßnahmen}

Gemäß Anlage 1 Teil B Absatz 2 Buchstabe e der IGV wird die Kapazität gefordert, für die Reaktion auf mögliche GNIT-Ereignisse empfohlene Maßnahmen zur
Befreiung von Insekten, zur Entrattung, zur Desinfektion, zur Entseuchung oder zur sonstigen Behandlung von Gepäck, Fracht, Containern, Beförderungsmitteln, Gütern oder Postpaketen anzuwenden, ggf. auch an Orten, die eigens für diesen Zweck bestimmt und ausgerüstet sind.

\section{Empfehlung:}

(s. auch $₫ 13$ Absatz 5 Satz 1 Nummer 5 IGV-DG):

Auf Anordnung der zuständigen Gesundheitsbehörde führt der jeweilige Pflichtige ggf. eine Desinsektion, Entrattung, Desinfektion, Dekontamination oder sonstige erforderliche Behandlung, gemäß den rechtlichen Bestimmungen und den allgemein anerkannten Regeln der Technik, durch.

Die Durchführung von Bekämpfungsmaßnahmen kann sich beziehen auf:

- Beförderungsmittel (insbesondere Schiffe und Fahrzeuge),

- Gebäude der Hafenanlagen,

- Freiflächen im Hafen,

- Gepäck,

- Container und Ladegüter.

Administrative Vorkehrungen und Vereinbarungen liegen vor, für

- die sichere Aufbewahrung, Dekontamination oder Vernichtung von Gegenständen in Abstimmung mit der zuständigen Behörde.

\subsubsection{Personal}

Administrative Vorkehrungen und Vereinbarungen liegen vor, für

- Zugang zu ausgebildetem und qualifiziertem Personal zur angemessenen und zeitnahen Durchführung von empfohlenen Maßnahmen (Reinigung, Desinsektion, Entrattung, Desinfektion, Dekontamination oder sonstige erforderliche Behandlung), in Abstimmung mit den zuständigen Behörden.

Die Maßnahmen sollen, soweit möglich, während der geplanten Schiffsliegedauer abgeschlossen werden, oder es soll mindestens eine abschließende Beurteilung über die notwendigen Maßnahmen erfolgen.

\subsubsection{Räumlichkeiten}

Administrative Vorkehrungen und Vereinbarungen liegen vor, für

- die sichere Aufbewahrung, Dekontamination oder Vernichtung von Gegenständen in Abstimmung mit der zuständigen Behörde.

\subsection{Medizinische Ein- und Ausreisekontrolle}

Gemäß Anlage 1 Teil B Absatz 2 Buchstabe f der IGV wird die Kapazität gefordert, für die Reaktion auf mögliche GNIT-Ereignisse Ein- oder Ausreisekontrollen für ankommende und abreisende Personen durchzuführen.

\section{Empfehlung:}

\subsubsection{Personal}

Administrative Vorkehrungen und Vereinbarungen liegen vor, für

- den 24/7 Zugang zu benanntem Personal im Hafen, das Schlüsselentscheidungen zur Umsetzung von Anordnungen der zuständigen Behörde trifft, koordiniert, implementiert und ggf. durchführt.

\subsubsection{Organisatorische Abwicklung}

Administrative Vorkehrungen und Vereinbarungen liegen vor, für

- die organisatorische Abwicklung von medizinischen Ein- und Ausreisekontrollen; und

- ein lageabhängiges, definiertes, dokumentiertes und bei allen Beteiligten bekanntes und trainiertes Verfahren der medizinischen Ein- und Ausreisekontrolle.

\subsection{Transfer von Reisenden, die möglicherweise infiziert oder verseucht sind}

Gemäß Anlage 1 Teil B Absatz 2 Buchstabe g der IGV wird die Kapazität gefordert, für die Reaktion auf mögliche GNIT-Ereignisse für den Transfer von Reisenden, die möglicherweise infiziert oder verseucht sind, Zugang zu eigens vorgesehenen Einrichtungen und zu ausgebildetem, mit geeigneten Schutzvorkehrungen versehenem Personal bereitzustellen. 
Empfehlung:

\subsubsection{Personal}

Administrative Vorkehrungen und Vereinbarungen liegen vor, für

- den 24/7 Zugang zu ausgebildetem und benanntem Personal zur Sicherstellung eines angemessenen Transfers von Reisenden, die möglicherweise infiziert oder verseucht sind zu eigens vorgesehenen Einrichtungen (z. B. Quarantäneeinrichtung).

\subsubsection{Ausrüstung}

Administrative Vorkehrungen und Vereinbarungen liegen vor, für

- die Vorhaltung der erforderlichen persönlichen Schutzausrüstung; und

- den 24/7 Zugang zu Fahrzeugen für den sicheren und hygienischen Transfer von Reisenden, die möglicherweise infiziert oder verseucht sind zu geeigneten Einrichtungen.

\section{Kontaktpersonen-Ermittlung}

Gemäß Artikel 18 Absatz 1 der IGV kann die WHO Empfehlungen an die Vertragsstaaten hinsichtlich der Ermittlung von Kontakten verdächtiger oder betroffener Personen geben.

\section{Empfehlung:}

(s. auch $\$ 17$ Absatz 3 i.V.m. $\$ 12$ IGVDG):

Administrative Vorkehrungen und Vereinbarungen liegen vor, für

- die zeitnahe Übermittlung der Passagierlisten mit Kontaktdaten durch die Schiffsführung oder den Reiseveranstalter an das zuständige $\mathrm{Ge}$ sundheitsamt gemäß den rechtlichen Bestimmungen ( $\$ 17$ Absatz 3 i.V.m. $\$ 12$ Absatz 5 IGV-DG);

- die Verteilung von Aussteigekarten (Passenger Locator Form, PLF), ggf. auf Anordnung des Gesundheitsamts ( $\$ 17$ Absatz 3 i.V.m. $\$ 12$ Absatz 3 IGV-DG); standardisierte Aussteigekarten gemäß IGV-DG Anlage 1a sind beim HÄD (ÖGD) vorzuhalten;

- ein definiertes, dokumentiertes und bei allen Beteiligten bekanntes und trainiertes Verfahren der Kontaktpersonen-Ermittlung; und
- benanntes, qualifiziertes Personal zur Bearbeitung, Digitalisierung, Ermittlung und Kontaktaufnahme.

Hinweis: Das Gesundheitsamt darf die gemäß der Infektionsschutzgesetze (IfSG) erhobenen personenbezogenen Daten nur für seine Aufgaben nach dem IfSG nutzen und ist insoweit für die Einhaltung des Datenschutzes verantwortlich.

\section{Ausbildung und Inübunghaltung}

Administrative Vorkehrungen und Vereinbarungen liegen vor, für

- ein Ausbildungs- und Fortbildungsprogramm für alle Einsatzbeteiligten;

- die Vertrautheit aller benannten Personen mit ihrem Einsatzbereich, den Verfahrensweisen, Dokumentationspflichten und Vorschriften, die im Rahmen praktischer Übungen nachgewiesen ist;

- die Geübtheit des Personals in der Benutzung von persönlicher Schutzausrüstung;

- ein definiertes, dokumentiertes und bei allen Beteiligten bekanntes und trainiertes Verfahren der Impfvorsorge bzw. Prä- bzw. Postexpositionsprophylaxe; und

- die Kenntnis der epidemiologischen Situation im Hafen (u. a. die Kenntnis der allgemeinen Risiken für die öffentliche Gesundheit, welche bei Routineuntersuchungen entdeckt werden, und Kenntnis der üblichen Risiken für die öffentliche Gesundheit, welche mit Art und Größe, Herkunft und Bestimmung der Schiffe assoziiert sind.).

\section{Erfahrungsaustausch und Fortentwicklung der Kernkapazitäten}

Ein regelmäßiger Erfahrungsaustausch (z.B. im Rahmen des AkKÜ) und eine kontinuierliche Fortentwicklung der Kernkapazitäten, die jederzeit verfügbar sein müssen bzw. der Kernkapazitäten, welche zur Reaktion auf mögliche gesundheitliche Notlagen internationaler Tragweite an Grenzübergangsstellen vorhanden sein sollen, sind sinnvoll.

\section{Literatur}

1. Gesetz zu den Internationalen Gesundheitsvorschriften (2005) (IGV) vom 23. Mai 2005, vom 20. Juli 2007 (BGBI. II, S. 930) https://www.rki. de/DE/Content/Infekt/IGV/Gesetz_IGV_de-en. pdf?_blob=publicationFile.

2. Gesetz zur Durchführung der Internationalen Gesundheitsvorschriften (2005) (IGV-Durchführungsgesetz - IGV-DG) vom 21. März 2013 (BGBI. IS. 566). http://www.gesetze-im-internet.de/ igv-dg/index.html.

3. Richtlinie 2010/65/EU des europäischen Parlamentes und des Rates vom 20. Oktober 2010 über Meldeformalitäten für Schiffe beim Einlaufen in und/oder Auslaufen aus Häfen der Mitgliedstaaten und zur Aufhebung der Richtlinie 2002/6/EG. 2010. Internet: http://eur-lex.europa.eu/LexUriServ/LexUriServ.do?uri=OJ:L:2010:283:0001:0010: DE:PDF.

4. Medizinproduktegesetz in der Fassung der Bekanntmachung vom 7. August 2002 (BGBI. IS. 3146), das durch Artikel 4 Absatz 62 des Gesetzes vom 7. August 2013 (BGBI. IS. 3154) geändert worden ist. http://www.gesetze-im-internet.de/ $\mathrm{mpg} /$.

5. Infektionsschutzgesetz vom 20. Juli 2000 (BGBI. I S. 1045). Internet: https://www.gesetze-im-internet. de/ifsg/.

6. Tiergesundheitsgesetz vom 22. Mai 2013 (BGBI. IS. 1324). Internet: https://www.gesetze-im-internet. de/tiergesg/.

7. Vorschlag für eine Verordnung des Europäischen Parlaments und des Rates über die Fahrgastrechte im See- und Binnenschiffsverkehr und zur Änderung der Verordnung (EG) Nr. 2006/2004 über die Zusammenarbeit zwischen den für die Durchsetzung der Verbraucherschutzgesetze zuständigen nationalen Behörden. 2009. Internet: http://register.consilium.europa.eu/pdf/de/09/ st13/st13874.de09.pdf. 